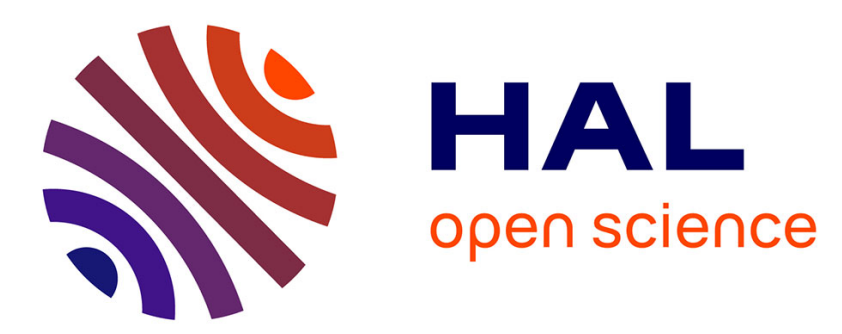

\title{
Altering the Stiffness, Friction, and Shape Perception of Tangible Objects in Virtual Reality Using Wearable Haptics
}

Steeven Villa Salazar, Claudio Pacchierotti, Xavier de Tinguy, Anderson Maciel, Maud Marchal

\section{To cite this version:}

Steeven Villa Salazar, Claudio Pacchierotti, Xavier de Tinguy, Anderson Maciel, Maud Marchal. Altering the Stiffness, Friction, and Shape Perception of Tangible Objects in Virtual Reality Using Wearable Haptics. IEEE Transactions on Haptics (ToH), 2020, 13 (1), pp.167-174. 10.1109/TOH.2020.2967389 . hal-02450301

\section{HAL Id: hal-02450301 \\ https://hal.inria.fr/hal-02450301}

Submitted on 22 Jan 2020

HAL is a multi-disciplinary open access archive for the deposit and dissemination of scientific research documents, whether they are published or not. The documents may come from teaching and research institutions in France or abroad, or from public or private research centers.
L'archive ouverte pluridisciplinaire HAL, est destinée au dépôt et à la diffusion de documents scientifiques de niveau recherche, publiés ou non, émanant des établissements d'enseignement et de recherche français ou étrangers, des laboratoires publics ou privés. 


\title{
Altering the Stiffness, Friction, and Shape Perception of Tangible Objects in Virtual Reality Using Wearable Haptics
}

\author{
Steeven Villa Salazar, Claudio Pacchierotti, Xavier de Tinguy, Anderson Maciel, Maud Marchal
}

\begin{abstract}
Tangible objects are used in Virtual Reality (VR) and Augmented Reality (AR) to enhance haptic information on the general shape of virtual objects. However, they are often passive or unable to simulate rich varying mechanical properties. This paper studies the effect of combining simple passive tangible objects and wearable haptics for improving the display of varying stiffness, friction, and shape sensations in these environments. By providing timely cutaneous stimuli through a wearable finger device, we can make an object feel softer or more slippery than it really is, and we can also create the illusion of encountering virtual bumps and holes. We evaluate the proposed approach carrying out three experiments with human subjects. Results confirm that we can increase the compliance of a tangible object by varying the pressure applied through a wearable device. We are also able to simulate the presence of bumps and holes by providing timely pressure and skin stretch sensations. Altering the friction of a tangible surface showed recognition rates above the chance level, albeit lower than those registered in the other experiments. Finally, we show the potential of our techniques in an immersive medical palpation use case in VR. These results pave the way for novel and promising haptic interactions in VR, better exploiting the multiple ways of providing simple, unobtrusive, and inexpensive haptic displays.
\end{abstract}

\section{INTRODUCTION}

O $\mathrm{NE}$ of the presence-breaking factors when immersed in an artificial world is the lack of haptic feedback [1]. Two approaches for addressing this need are tangible objects and wearable haptics.

Tangible objects have proven to be effective at conveying haptic information about distributed shape and weight, improving the immersiveness of Virtual Reality (VR) and Augmented Reality (AR) systems even when providing no additional haptic feedback [2], [3]. For example, Insko [2] presented a set of inexpensive tangible objects for augmenting virtual environments. One of his studies investigated the effects of augmenting a visual-cliff environment with a slight tangible ledge. Participants exhibited more behaviors associated with pit avoidance when interacting with the physical ledge than when not. Applications of tangible objects in VR and AR generally show a consistent immersion increase across multiple scenarios, users, and objects. However, in most cases, the tangible objects used are passive and made of relatively inexpensive materials, and they are thus not able to actively change their mechanical properties, e.g., their shape, texture, or weight. To address this issue, researchers worked on actuated tangible objects, capable of actively changing their characteristics as users interact with them [4]. Although rather effective, developing multiple, ad-hoc, haptic-enabled tangible objects for each scenario requires a significant amount of work. Moreover, actuated tangible objects can be expensive and complex to build.

Wearable haptics has also been proven effective at conveying haptic sensations in a comfortable and unobtrusive manner [5]-[12]. For example, Leonardis et al. [7] used two wearable fingertip devices in a pick-and-place experiment in VR. Each device moved a rigid tactor in contact with the skin, providing skin stretch and making/breaking contact sensations. Schorr and Okamura [6] developed a wearable

This research has received funding from the EU H2020 research and innovation programme (grant agreement No 801413, project "H-Reality") and Fapergs-Brazil PqG 2017 proj CHIHC.

S. Villa is with Univ Rennes, CNRS, Inria, IRISA - Rennes, FR; with Federal Univ. of Rio Grande do Sul - Porto Alegre, BR; and with Ludwig Maximilian University - Munich, DE. steeven.villa@um.ifi.lmu.de

C. Pacchierotti is with Univ Rennes, CNRS, Inria, IRISA - Rennes, FR.

A. Maciel is with Federal Univ. of Rio Grande do Sul - Porto Alegre, BR.

$\mathrm{X}$. de Tinguy and $\mathrm{M}$. Marchal are with Univ Rennes, INSA, IRISA, Inria, CNRS - Rennes, FR.

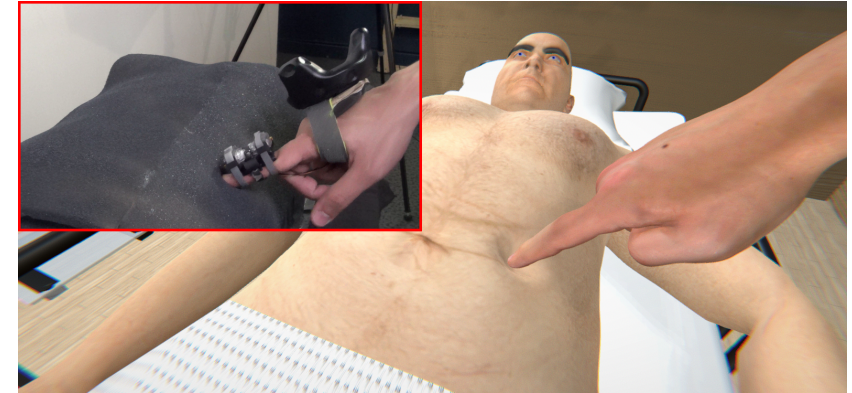

Fig. 1. Application example. A human user wearing a finger device interacts with a tangible object that resembles the abdomen of a virtual human patient. Providing timely cutaneous stimuli via the wearable haptic device, we can alter the stiffness and shape perception of passive tangible objects. For example, in the context of medical palpation, we can simulate the presence of a tender body part or of a small bump representing a cyst.

device able to make and break contact in addition to rendering shear and normal skin deformation to the finger pad. Although rather popular, these wearable interfaces only provide cutaneous stimulation, and they are thus not able to simulate stiff contacts or provide information about distributed shape. Hand-held haptics solutions for VR also exist, but they significantly limit the user's capability of directly interacting with a tangible environment [13], [14].

This paper aims at enhancing haptic rendering in virtual environments by taking the best of the two solutions discussed above. While passive tangible objects are effective at providing global and distributed shape sensations, they are unable to simulate rich varying haptic sensations. Conversely, wearable cutaneous haptics can unobtrusively display varying haptic sensations, but they are unable to provide elaborated kinesthetic stimuli, thus failing at simulating stiff contacts and global shapes. Other attempts at enhancing the perception of tangible objects via simple means exist, but none of them has been proven capable of providing well-rounded sensations in such an unobtrusive way. One of these alternative approaches is pseudohaptics, which uses vision to distort haptic perception. It has been employed to alter haptic properties such as the stiffness of a virtual spring, the texture of an image, or the mass of a virtual object [15], [16]. However, as pseudohaptics does not provide any haptic feedback, its augmentation capabilities are limited. Another interesting approach is interacting with tangible objects through external haptic tools [17]-[19]. Harders et al. [17] developed an AR system augmenting a leg dummy with virtual soft tissue. Users interacted with the dummy through a PHANToM end-effector, feeling the combination of haptic sensations due to the contact with the dummy and the additional feedback provided by the haptic interface. More recently, Culbertson and Kuchenbecker [18] used an ungrounded haptic system that alters the roughness and friction of a rigid tangible object. Users interact with the object via a haptic-enabled stylus, that actuates a vibration waveform through an embedded voice coil. Although effective, these approaches make use of external interfaces, which mediate the interaction between the user and the tangible object. Non-mediated solutions are very rare. Three of the very few examples uses a wearable vibrotactile unit on the finger to alter the roughness of tangible rigid surfaces [20]-[22].

In this paper, we present three perceptual studies analyzing the capability of wearable cutaneous stimuli to alter the stiffness, friction, and shape perception of tangible objects. By providing timely cuta- 


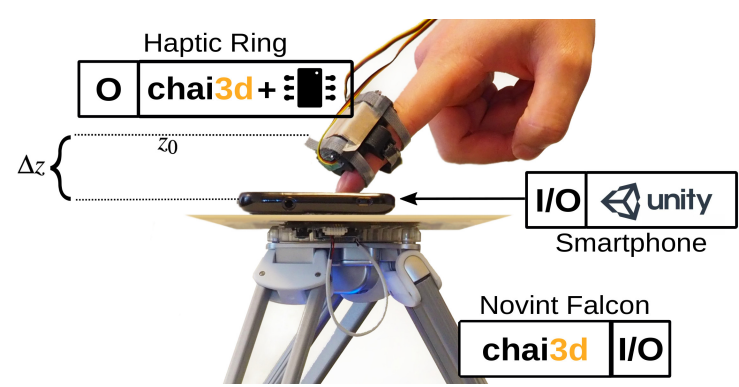

(a) Experimental setup

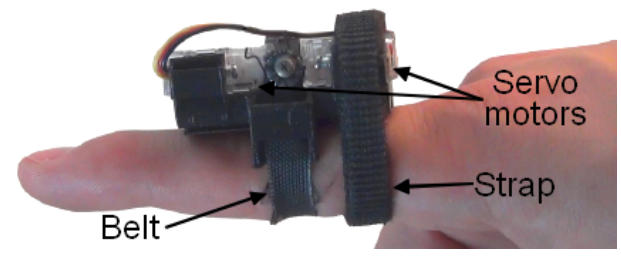

(b) Wearable cutaneous interface for the finger (hRing)

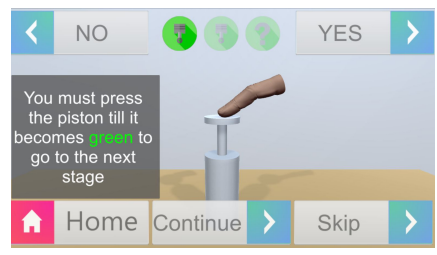

(c) User interface for Exp. \#1.

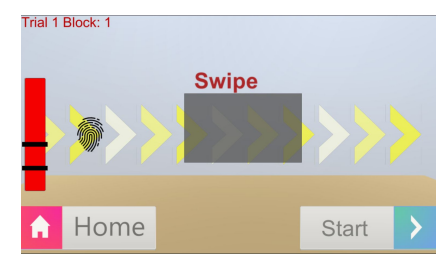

(d) User interface for Exps. \#2 and \#3.
Fig. 2. Experimental setup. It comprises a grounded haptic interface simulating the tangible object (Novint Falcon), a wearable cutaneous device for the finger, and a smartphone that tracks the interaction point and guide the user via a Unity3D user interface. We used the grounded interface instead of a passive tangible object to be able to easily change the object's mechanical properties and control the applied force.

neous stimuli through a wearable finger interface, we aim at making an object feel softer or more slippery than it really is and at creating the illusion of encountering virtual bumps and holes. This approach can be useful in VR and AR environments to alter at runtime the perceived mechanical properties of passive and inexpensive tangible objects. We evaluate the proposed approach via three human subjects studies, and we also present a representative use case in a VR medical palpation task. We started to research this topic in the preliminary work of [23], where we used a wearable interface to increase the perceived stiffness of a passive tangible object. In this work, we significantly extend the approach to consider again the perception of stiffness together with that of friction and shape, as well as a new extensive evaluation and use case. Our objective is to understand which environmental features can be altered via wearable cutaneous stimulation, as a starting point for a deeper analysis and understanding of the potential of combining wearable haptics and tangible objects in VR and AR. A video presenting the work can be found as supplemental material and at https://youtu.be/3CSqcOUeUeA.

\section{EXPERIMENTAL APPARATUS}

We devised a haptic system composed of a Novint Falcon grounded interface, a wearable cutaneous device for the fingertip, and a Unityready smartphone. The setup is shown in Fig. 2a. The smartphone was secured to a flat plastic surface attached to the end-effector of the Falcon interface, which was in turn placed on a table with its end-effector facing upwards. Users were asked to wear the cutaneous device on the middle phalanx of their index finger, so as to leave the fingertip free to touch the smartphone touchscreen. Using this device at the middle phalanx has been already proven effective for stiffness altering in [23]. The device is capable of providing pressure and skin stretch stimuli. It has been inspired by a similar ring-like device

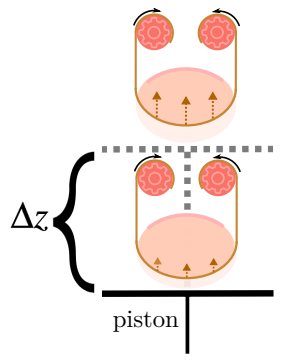

(a) Experiment \#1: altering the perception of stiffness

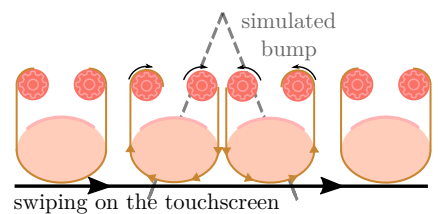

(c) Experiment \#2: bump rendered with a varying skin stretch

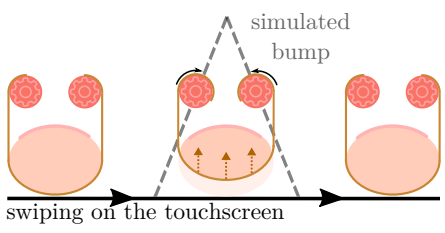

(b) Experiment \#2: bump rendered with a varying pressure

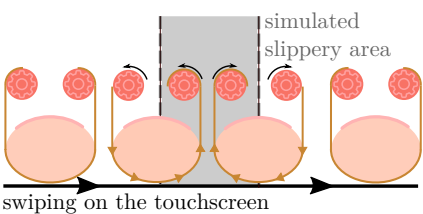

(d) Experiment \#3: sticky area rendered with a varying skin stretch
Fig. 3. The four haptic rendering approaches using the wearable interface for altering the (a) stiffness, (b),(c) shape, and (d) friction of tangible objects.

presented in [24], called "hRing" (see Fig. 2b). It is composed of two servo motors and a fabric belt, that applies the requested stimuli to the skin. A Velcro strap band is used to secure the device on the finger. When the two motors rotate in opposite directions, the belt is pulled up or down, providing a varying force normal to the finger (pressure); when the two motors spin in the same direction, the belt applies a shear force to the finger (skin stretch). The maximum displacement range of the device is $6 \mathrm{~mm}$. Considering an isotropic elastic behavior of the skin with elastic constant $0.5 \mathrm{~N} / \mathrm{mm}$ [9], [24], the device can apply a maximum force of $3 \mathrm{~N}$.

\section{EXPERIMENT \#1: ALTERING THE PERCEIVED STIFFNESS}

The objective of this first experiment is to evaluate the capability of wearable cutaneous stimuli to alter the stiffness perception of tangible objects, making them feel softer than they really are. In [23], we carried out a complementary evaluation. By providing timely cutaneous pressure sensations through a wearable device, it was possible to increase the perceived stiffness of passive tangible objects. Moreover, we proved that it was not necessary to provide the additional cutaneous sensation exactly where the contact with the tangible object happened (i.e., fingertip). Instead, the cutaneous stimulation could be provided on the middle or proximal finger phalanx with no significant degradation of the perceptual effect. By wearing the device on the middle phalanx, we also leave the fingertip free to directly interact with the tangible object.

In this paper, we evaluated if it was possible to decrease the perceived stiffness of tangibles by providing timely additional cutaneous pressure sensations. Starting with a compressed fingertip, we tested if reducing the pressure applied whenever the user contacts the tangible object can decrease its perceived stiffness. We believe this effect to be possible as humans are more receptive to changes in haptic stimulation rather than to its absolute value [25].

Participants: Fourteen healthy participants took part in this experiment (13 males, 1 female; age $\mathrm{M}=29.4, \mathrm{SD}=7.5$ ).

Experimental Procedure: Participants had to compare two pistons with different rendered stiffness, modeled by a $1 \mathrm{D}$ spring law: $f=-k \Delta z$ if $\Delta z>0 \mathrm{~mm}, 0 \mathrm{~N}$ otherwise, where $\Delta z$ is the difference between the position of the Falcon's end-effector and the resting position of the piston. Subjects were asked to press down a first piston until the screen told them to stop, and then move their fingertip away from the end-effector to enable its release (see Fig. 2c). After that, they were asked to interact in a similar way with a second piston. Finally, users had to judge if piston \#1 felt stiffer than 
piston \#2. One piston served as a reference, displaying a reference stiffness $k_{r e f, F}=0.1 \mathrm{~N} / \mathrm{mm}$ provided by the Falcon and a constant high pressure provided by the hRing. The other piston displayed a variable stiffness $k_{t e s t, F}-k_{\text {test }, T}$ provided using the Falcon and the hRing device, respectively. The Falcon provides haptic feedback as expected, increasing the force provided as the user presses the springmodeled piston (and $\Delta z$ increases). The test stiffness $k_{t e s t . F}$ provided by the Falcon was changed between the trials. In this experiment, our objective was to modify the user perception by changing the behavior of the hRing. For that purpose, we started each trial by applying a high pressure on the fingertip, which was then decreased as the user presses the piston (see Fig. 3a). We have highlighted this behavior by marking the contribution of the Falcon, $k_{t e s t, F}$, as a positive value, and the contribution of the hRing, $-k_{\text {test }, T}$, as a negative value.

Experimental Design: Similarly to [23], we considered 7 values of stiffness $k_{\text {test }, F}$ to be compared with the stiffness of the reference piston $k_{\text {ref }}=0.1 \mathrm{~N} / \mathrm{mm}$. The seven values of the test piston were: $-92.3 \%,-61.5 \%,-23 \%,+0 \%,+23 \%,+61.5 \%$, and $+92.3 \%$ of the reference stiffness. The hRing always rendered the same "negative" stiffness $-k_{t e s t, T}$ at the beginning of each trial. We then compared two experimental conditions for the hRing behavior:

- C1: the hRing diminishes the pressure applied as the user presses the piston.

- C2: the hRing applies a constant pressure during the interaction.

Participants were presented with 70 trials, divided in 10 blocks of 7 trials. 5 blocks were achieved with condition $\mathbf{C 1}$ while the 5 others were with $\mathbf{C 2}$. The order was randomized between the blocks. Each block presented a set of couples of pistons made of 7 different stiffness values. The order of presentation of the two pistons was counterbalanced to avoid any order effect.

Collected Data: For each couple of pistons, we collected the participant's answer. It corresponds to the piston that was reported as the stiffest. The measure was then collected as a true discovery rate, i.e., if the answer corresponds to the stiffest value rendered. Participants also completed a subjective questionnaire at the end of the experiment. Each question was answered using a 7-item Likert scale: Q1. The haptic device on your finger contributed to the perception of stiffness; Q2. The combination of both cutaneous and kinesthetic sensations contributed to the perception of stiffness; Q3. The action of the tactile device affects my perception of stiffness; $\mathbf{Q 4}$. Practicing improves the association of both cutaneous and kinesthetic sensations; Q5. After the experiment, I felt tired; Q6. The wearable device in my finger distracted me from the primary task.

Results: We used a logistic regression model for the recognition rate of the stiffest piston for two independent conditions of the experimental design. We performed a one-way repeated-measures ANOVA to validate our hypothesis that diminishing wearable cutaneous stimuli while pressing the piston decreases the stiffness perception of the tangible object. We found a significant difference between conditions $(F(1,21)=19.66, p<0.001)$, which let us reject the null hypothesis (for al pha $=0.005$ ). Point of Subjective Equality (PSE) for C2 was $-5.8 \%(0.094 \mathrm{~N} / \mathrm{mm})$ while for $\mathbf{C 1} 3.6 \%(0.103 \mathrm{~N} / \mathrm{mm})$. In the same way, Just Noticeable Differences were $19.8 \%$ and $20.1 \%$ for $\mathbf{C 1}$ and C2 respectively. The magnitude of these just noticeable values is coherent with previous studies [23], [26].

Figure 4 shows the effect of $\mathbf{C 2}$ : a visible offset to the left (less stiffness) is present in C2. This reflects the influence of the tactile stimuli on the stiffness discrimination. The presence of a pre-load of the wearable device when not touching and then a release of the device as long as the user presses reduces the perceived stiffness, in other words, the difference of pressure and not the actual pressure directly impacts on the participant's perception. Also, the difference of almost $10 \%$ between Points of Subjective Equality gives cues about the capability of this rendering mode.

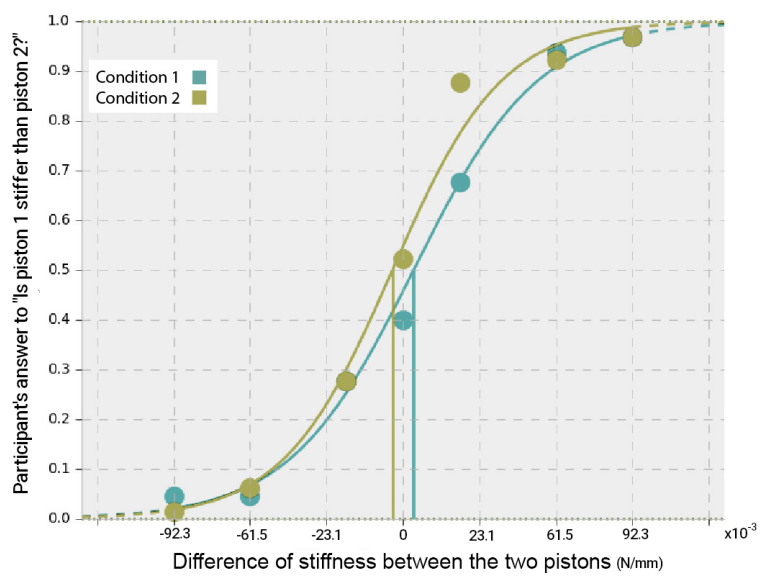

Fig. 4. Recognition rate for experiment \#1. The PSE of the releasing condition C2 (in yellow) is offset regarding the constant pressure condition C1 (in blue).

Additionally, the thresholds of perceived force versus recognition rate also reflects a shift in the perceived stiffness. There is a constant offset of $M=8.93 \%$ ( $S D=0.05 \%$ ) between the curves $(8.9 \%$ at $25 \%$, $9.4 \%$ at $50 \%$, and $8.4 \%$ at $75 \%$ ) while the discrimination sensitivity (curve steepness) remains similar (39.7\% for $\mathbf{C 1}$ and $40.1 \%$ for C2). This shows that tactile stimuli impact mostly the stiffness perceived, but not the accuracy or overall performance of the subjects. In this way, the use of the wearable device rendering does not decrease the capacity of the users to discriminate between stiffnesses.

Fig. 5 summarizes the answers to the questionnaire (7-point Likert scale). The participants found that the device barely contributed to the perception of stiffness $(\mathrm{Q} 1, M=4.0 S D=2.0)$. However, most of them agree that the combination of both feedbacks contributed somehow to the perception of stiffness $(\mathrm{Q} 2, M=5.0 S D=1.7)$. Also, the tactile device barely affected their perception of stiffness (Q3, $M=4.6 S D=1.9$ ). Almost all of them agree that practicing improves the association of both sensations $(\mathrm{Q} 4, M=5.7 S D=0.9)$. The device had not really impacted on their fatigue since users reported not feeling tired (Q5, $M=3.1 S D=1.6)$. Finally, subjects answered they were distracted by the actuation of our device $(\mathrm{Q} 6, M=3.5 S D=2.1)$

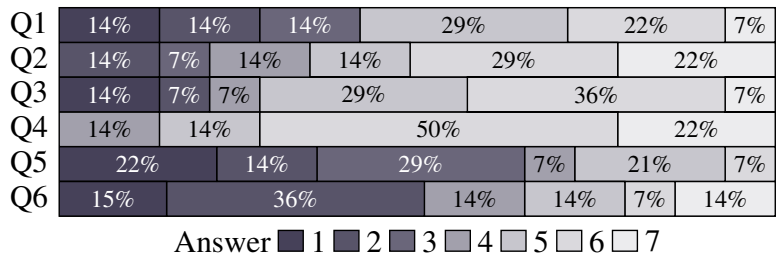

Fig. 5. Experiment \#1. Total answers to the subjective questionnaire.

Discussion: Results suggest that our hypothesis about altering stiffness rendering is correct. Indeed, the curves describing the recognition rate clearly show an effect of using the tactile device. Whenever the device was diminishing its pressure $(\mathbf{C 1})$, users perceived the piston softer than it really was. This result confirms the understanding that humans are more receptive to changes in haptic stimulation rather than to its absolute value [25]. However, it is the first time that this effect is demonstrated for changing the properties of objects in VR. Of course, this effect only works if the device pre-compresses the user's finger for a certain amount of time before its release, limiting the frequency below which this effect can be exploited. This issue did not affect the preliminary and somewhat complementary work of de Tinguy et al. [23], where the authors increased the perceived stiffness of a tangible object by applying timely additional stimuli via the same wearable device. In that case, the effect was 
elicited by simply applying more pressure onto the user's finger, with no limitation in terms of frequency. In our case, it would be very interesting to understand the effect of the pre-compression time on our stiffness alteration effect. In other words, we would like to evaluate the minimum time the device needs to pre-compress the finger skin while still being able to elicit the stiffness alteration effect shown in Fig. 4. Another approach could be to compress the finger skin at a significantly lower speed than the release action, so that users would again feel more the release of the belt and almost neglect its slow compression. This second approach reminds the one used for inducing ungrounded pulling sensations using asymmetric vibrations [27]. Asymmetric vibrations are characterized by large positive acceleration peaks and small negative acceleration peaks, so that the user mostly feels the large positive ones. Finally, we highlight that the stiffness of the piston was set using the standard APIs of the Novint Falcon. A better approach would be to instrument the Falcon's end-effector with a force sensor, and use that information to drive the interface using a closed-loop force control approach. Nonetheless, in both $\mathbf{C 1}$ and $\mathbf{C 2}$, the Falcon was driven in the same way, proving that the effect of the wearable haptic stimulation is still valid.

\section{EXPERIMENT \#2: ENHANCING THE PERCEPTION OF SHAPE}

The objective of the second experiment is to evaluate the capability of wearable cutaneous stimuli to enhance the shape perception of tangible objects. Specifically, by applying timely cutaneous stimulation, we study whether it is possible to create the illusion of touching bumps and holes while interacting with a flat tangible surface. We considered bumps and holes having a width of $3 \mathrm{~cm}$.

Participants: Fourteen healthy participants took part in this experiment (13 males, 1 female; age $M=24, S D=3.3$ ). Nine of them also participated in Experiment \#1.

Experimental Procedure: Participants had to choose whether they were feeling a bump or a hole while swiping the flat surface of the smartphone $(12 \mathrm{~cm})$, following a 2-AFC (2-Alternative Forced Choice) protocol. Participants were not trained on how a bump/hole felt; they had to autonomously relate the received stimuli with either a bump or a hole. Subjects were asked to touch one side of the screen and then swipe toward the opposite side, following a fingerprint moving on the screen (see Fig. 2d). To ensure that all subjects apply the same force to the screen, we showed the applied normal force on the touchscreen and asked them to keep it in a target range (vertical bar on the left of Fig. 2d, it becomes green when the force applied is in the target range). The Falcon device here is only used to control the normal force and does not provide any force feedback.

We considered two different ways of rendering the bumps and holes. In the first one, we used varying pressure stimuli. As shown in Fig. 3b, the bump is modeled as a triangular prism and the wearable device simulates its presence by providing a varying normal pressure: medium when the finger is far from the bump $(1.3 \mathrm{~N})$ and maximum when it is at its top $(3 \mathrm{~N})$. The hole is modeled as a reverse triangular prism and the wearable device simulates its presence by providing again a varying normal pressure: medium when the finger is far from the bump $(1.3 \mathrm{~N})$ and minimum when it is at its bottom $(0.4 \mathrm{~N})$. In the second rendering approach, we used varying skin stretch stimuli. As shown in Fig. 3c, the bump is again modeled as a triangular prism and the wearable device simulates its presence by providing a varying skin stretch: opposite to the finger's motion when ascending the bump and toward the finger's motion when descending the bump. Similarly, a hole is rendered by providing a skin stretch toward the finger's motion when descending to the hole and opposite to the finger's motion when ascending from the hole.

Experimental Design: Following the two ways of rendering bumps and holes, we considered two experimental conditions:

- C1: Bumps and holes rendered via varying pressure.
- C2: Bumps and holes rendered via varying skin stretch.

Participants were presented with 40 trials, divided in 2 blocks of 20 trials, one condition per block, in counter-balanced order. To avoid any crossmodal effect, we changed the swiping direction randomly so as to have an equal amount of swipes in each direction.

Collected Data: For each swipe, we collected the participant's answer ("bump" or "hole"). Participants also completed a subjective questionnaire at the end of each condition. Each question was answered using a 7-item Likert scale: Q1. When swiping, I felt the bumps and holes; Q2. When swiping, it was easy to discriminate the shapes; Q3. It was easier to perceive... 1. Bump - 7. Hole; Q4. The wearable device distracted me from the primary task.

Results: For this experiment, we stored the answers of the users and contrasted them with the rendered stimuli. Figure 6 shows the results of the two conditions. The first condition $(\mathbf{C 1}$, rendering via pressure) outperforms the second condition $(\mathbf{C} 2$, rendering via skin stretch) for rendering holes. However, for the case of the bumps, there is a smaller difference.
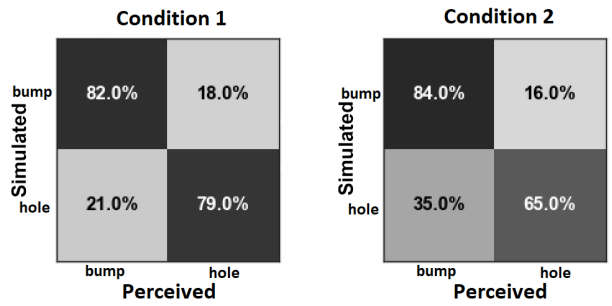

Fig. 6. Experiment \#2. Confusion matrices for the two ways of rendering bumps and holes: C1 by varying the pressure on the finger, C2 by varying the skin stretch on the finger.

Table I below gives additional information about the performance obtained by the subjects in each condition. The best overall accuracy was obtained with C1. Also in Precision and Recall, C1 performed better. We calculated the accuracy dividing the sum of correct answers by the sum of all the answers. The error rate is the sum of the wrong answers divided by all the answers. Precision is the number of shapes correctly classified (bumps or holes) divided by the total number of shapes predicted. The recall is the number of shapes correctly classified divided by the number of shapes rendered.

TABLE I

EXPERIMENT \#2. RESULTS. P = PRECISION, R = RECALL

\begin{tabular}{c|ccccccc} 
& $\begin{array}{c}\text { Accu- } \\
\text { racy } \\
(\%)\end{array}$ & $\begin{array}{c}\text { Error } \\
\text { Rate } \\
(\%)\end{array}$ & $\begin{array}{c}\mathrm{P} \\
\text { Bumps }\end{array}$ & $\begin{array}{c}\mathrm{R} \\
\text { Bumps } \\
(\%)\end{array}$ & $\begin{array}{c}\mathrm{P} \\
\text { Holes } \\
(\%)\end{array}$ & $\begin{array}{c}\mathrm{R} \\
\text { Holes }\end{array}$ & MCC \\
& 80.8 & 19.2 & 82.0 & 79.6 & 79.0 & 81.4 & 0.623 \\
C1 & 74.6 & 25.4 & 84.0 & 70.5 & 65.0 & 80.2 & 0.492
\end{tabular}

A revealing metric is the Matthews correlation coefficient (MCC) which is related to the chi-square statistic for a confusion matrix: the values range from -1 to 1 , being -1 a behavior opposed to the expected, 0 a random behavior and 1 a perfect classification. C1 obtained the best results $(M C C=0.623)$, which indicates a positive correlation between rendered and perceived stimulus. Besides, C2 shows a positive rendered-predicted stimulus but in a smaller amount, so, for the case of bumps and holes, the stimulation of slow adapting receptors instead of fast adapting receptors performed better. Paired t-tests revealed no statistically significant difference between the two conditions in any of the metrics $(p>0.05)$.

From the answers to the subjective questionnaire, users were more confident in C1 (Q1 $M=4.71 S D=1.54)$ than C2 (Q1 $M=4.00$ $S D=1.96)$. Also $\mathbf{C 1}$ performed better when discriminating shapes (being aware that the shape is different) $(\mathrm{Q} 2 M=4.92 S D=1.54)$ than C2 (Q2 $M=4.00 S D=1.92)$. When it comes to a specific shape, both rendering methods were evaluated very similar: bumps were easier to perceive than holes C1 (Q3 $M=2.64 S D=1.44), \mathbf{C 2}$ 
(Q3 $M=2.5 S D=1.34)$. Regarding how much the device impacted over the task in terms of distraction, users perceived that it was a bit more distractive in $\mathbf{C 1}(\mathrm{Q} 4 M=3.42 S D=1.60)$ than $\mathbf{C 2}(\mathrm{Q} 4 M=$ $3.07 S D=1.49)$. Paired t-tests revealed no statistically significant difference between the answers $(p>0.05)$.

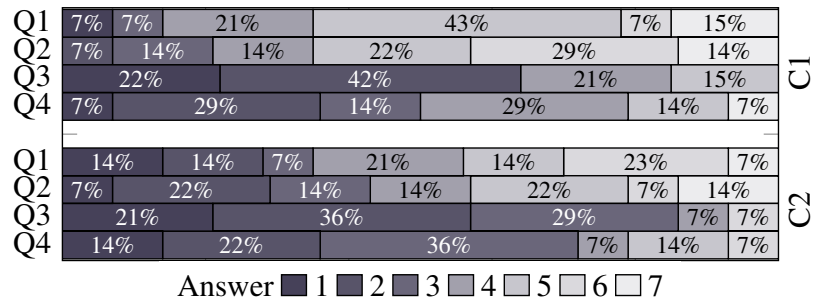

Fig. 7. Experiment \#2. Total answers of the subjective questionnaire.

Discussion: Results show that both proposed rendering techniques worked quite well in simulating the presence of bumps and holes on a flat tangible surface. As clear from the confusion matrices of Fig. 6, both approaches worked remarkably well in rendering bumps. However, C1 (rendering with pressure) worked better than C2 (rendering with skin stretch) when simulating the presence of holes. When a finger interacts with a real bump on a surface, the skin experiences a combination of our rendering techniques: higher pressure and skin stretch against the finger's motion when moving up the bump, lower pressure and skin stretch toward the finger's motion when moving down the bump. For this reason, it does not come as a surprise that both techniques achieve satisfactory performance. However, this result is still very relevant, as it is the first time that wearable haptics is used to alter the perception of shape in tangible objects. The degradation of performance in rendering holes in C2 comes as a surprise, especially when coupled with the good performance that the same condition has shown in rendering bumps (84\%, the highest performance registered). Playing with the medium pressure to vary the pressure amplitude could be a solution to improve the hole rendering. More experiments are needed to investigate this discrepancy. The registered users experience may come to our aid. In fact, users felt generally more confident in condition $\mathbf{C} 1$ than in C2. Some subjects also reported that discrimination of bumps was straightforward in both conditions. On the other hand, they could not always understand the rendering of holes. Finally, three subjects reported that being provided with the stimuli at the middle phalanx instead of the fingertip felt "strange" and "surprising," as they expected to receive the stimuli at the point of interaction with the tangible surface.

\section{EXPERIMENT \#3: MODIFYING THE PERCEPTION OF FRICTION}

The objective of the third experiment is to evaluate the capability of wearable cutaneous stimuli to modify the friction perception of tangible objects. Specifically, by applying timely skin stretch stimulation, we study if it is possible to make a tangible object feel more/less slippery than it really is.

Participants: The same fourteen participants who participated in Experiment \#2, also participated to this one.

Experimental Procedure: Experiments \#2 and \#3 were carried out in the same session, as two independent blocks. In this case, participants had to compare the friction on two surfaces. Similarly to Experiment \#2, subjects touched one side of the screen and then swiped toward the opposite side $(12 \mathrm{~cm})$, following a fingerprint moving on the screen (see Fig. 2d). They were asked to perform this interaction two times. Then, they were asked to judge if the first surface felt more slippery than the second one. Of course, differently from Experiment \#1, here we cannot change the true mechanical property of the tangible object, i.e., its friction. To simulate a slippery patch (grey zone in Fig. 2d) on the touchscreen, we used the hRing to provide skin stretch toward the finger's motion (see Fig. 3d). In the contrary, to simulate a sticky patch, the hRing provided skin stretch opposite to the finger's motion (similarly to $\mathbf{C} 2$ in Sec. IV).

Experimental Design: We considered one condition C1: slippery and sticky surface are rendered by varying skin stretch. Participants were presented with 20 trials, each trial composed of two swipes. To avoid any cross-modal effect, we changed the swiping direction randomly so as to have an equal amount of swipes in each direction.

Collected Data: For each trial, we collected the participant's answer ("Yes" or "No") to the the question is surface 1 slippery than surface 2?. Participants also completed a subjective questionnaire at the end of the experiment. Each question was answered using a 7-item Likert scale: Q1. When swiping, I felt the sticky and slippery surfaces; Q2. When swiping, it was easy to discriminate the surfaces; Q3. It was easier to perceive... 1. sticky - 7. slippery; Q4. The wearable device distracted me from the primary task.

Results: This experiment uses the same rendering as Sec. IVC2 (skin stretch), but focuses on simulating the presence of either a sticky or slippery area on the tangible surface. Figure $8 \mathrm{~b}$ shows the confusion matrix with the recognition rates above the chance level. The overall average accuracy $(61.2 \%)$, error rate $(38.8 \%)$, precision $(58.9 \%)$, negative predictive value $(63.0 \%)$, sensitivity $(60.5 \%)$, recall $(63.1 \%)$, and MCC $(0.288 \%)$ confirm a satisfactory performance, although lower than that registered when simulating bumps/holes using the same rendering approach.

Figure 8 a shows the answers to the subjective questionnaire. Users were not always convinced about the presence of a sticky or slippery surface on the tangible surface (Q1 $M=3.42 S D=1.39)$. Similarly, it was not found easy to discriminate between the two simulated surface properties $(\mathrm{Q} 2 M=3.00 S D=1.17)$ and that one was not easier to recognize than the other $(\mathrm{Q} 1 M=3.71 S D=1.58)$. Interestingly, they found the device to be more distractive in this experiment (Q1 $M=4.14 S D=1.79$ ) with respect to Sec. IV-C2.

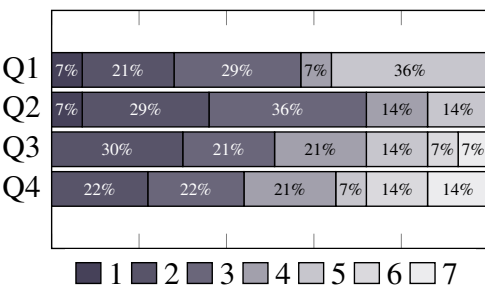

(a) Subjective data

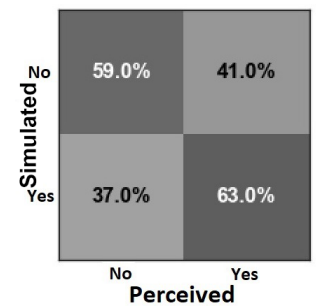

(b) Discrimination rates
Fig. 8. Experiment \#3. Total answers for the user's questionnaire and confusion matrix for the question is surface 1 slippery than surface 2?

Discussion: Results show that the proposed rendering technique provided recognition rate above the chance level. However, its performance is lower than that registered in Exp. \#2, when we used the same skin stretch approach to simulate the presence of bumps and holes. This came a bit as a surprise, as we believed that this rendering technique would stimulate the skin in a way that resembles a bit what happens in reality. For example, Provancher and Sylvester [28] found that the perceived magnitude of friction rendered by traditional force feedback can be increased through the addition of fingertip skin stretch. Instead, in this experiment, the performance of the task degraded significantly, and users also reported lower confidence in this condition w.r.t. to the previous ones. This can be perhaps explained from how humans perceive friction, which is related to the stickiness of the fingertip w.r.t. the surface as well as its velocity of motion. For this reason, there is a weaker correspondence between the skin stretch provided and how usually humans experience friction.

\section{USE CASE}

We designed a use case in VR considering a medical palpation scenario, illustrated in Fig. 1 and at https://youtu.be/3CSqcOUeUeA. 
The Unity3D scene is composed of a patient laying on a table inside a medical examination room, rendered to the human user via an HTC VIVE system. The human user wears one wearable cutaneous device on the middle phalanx of the right index finger. In front of the user, we place a large parallelepiped made of foam, which coarsely resembles the abdomen of the patient. The tangible object does not include any active part and it always shows the same mechanical properties. The position of the virtual hand avatar in the scene with respect of the body is carefully adjusted to match the position of the human hand with respect to the tangible abdomen. The user has to palpate the abdomen and locate a virtual cyst, simulated by a 3-cm-wide bump as described in Sec. IV-C2, and a tender zone, simulated by a 3-cmwide softer zone as described in Sec. III-C1.

\section{CONCLUSIONS AND FUTURE WORK}

In this paper, we presented an innovative approach for enhancing haptic sensations in VR and AR immersive environments. We proposed to combine the haptic capabilities of tangible objects and wearable haptics to modify the stiffness, friction, and shape perception of tangible objects. Whenever the user interacts with a tangible object in VR, the wearable interface provides a variable pressure or skin stretch stimulation, making the tangible object feel more/less stiff, more/less slippery, or having bumps/holes, depending on what is happening in the virtual scene. We used a wearable haptic device at the level of the middle finger phalanx so as to leave the user's fingertip free to directly interact with the tangible environment.

We carried out three human subject studies, evaluating the capability of altering the perception of stiffness (Exp. \#1), shape (Exp. \#2), and friction (Exp. \#3). Results showed that we can well alter the perception of stiffness by varying the pressure applied by the wearable device. We were also able to well simulate the presence of bumps and holes by providing timely pressure and skin stretch sensations. Altering the friction of a tangible surface showed recognition rates above the chance level, albeit lower than those registered in the other experiments. Finally, a use case showed the potential of this approach in an immersive VR medical scenario. These results pave the way for innovative haptic approaches in VR/AR, whose objective is to better exploit the many new haptic technologies providing haptic sensations in a simple and inexpensive way.

As for future work, we plan to extend the proposed approach to other types of haptic sensations and illusions, such as for simulating other types of shapes. Moreover, we plan to more deeply analyze the two first perceptual studies, which proved to be more promising. For example, we want to study the effect of the pre-compression time on the stiffness alteration effect and that of the starting pressure when rendering bumps and holes. Moreover, we plan to instrument the end-effector of the grounded haptic interface with a force sensor to precisely control the provided stiffness sensations. Finally, it is important to understand whether the cutaneous stimuli provided really elicit altered sensations of stiffness, shape, and friction, or if users simply learn to associate the action of the wearable device with an altered sensation of these target features. In other words, we want to understand to what extent would subjects still find the hapticallyaugmented tangible object altered (e.g., stiffer) if we did not ask the corresponding question (e.g., "which piston feels stiffer"?).

\section{REFERENCES}

[1] S. Lee and G. J. Kim, "Effects of haptic feedback, stereoscopy, and image resolution on performance and presence in remote navigation," Int. J. Human-Computer Studies, vol. 66, pp. 701-717, 2008.

[2] B. Insko, "Passive haptics significantly enhances virtual environments," Ph.D. dissertation, University of North Carolina at Chapel Hill, 2001.

[3] A. D. Cheok, X. Yang, Z. Z. Ying, M. Billinghurst, and H. Kato, "Touchspace: Mixed reality game space based on ubiquitous, tangible, and social computing," Personal and ubiquitous computing, vol. 6, no. 56, pp. 430-442, 2002.
[4] H. Ishii, D. Leithinger, S. Follmer, A. Zoran, P. Schoessler, and J. Counts, "Transform: Embodiment of radical atoms at milano design week," in Proc. CHI, 2015, pp. 687-694.

[5] C. Pacchierotti, S. Sinclair, M. Solazzi, A. Frisoli, V. Hayward, and D. Prattichizzo, "Wearable haptic systems for the fingertip and the hand: taxonomy, review, and perspectives," IEEE Trans. Haptics, vol. 10, no. 4, pp. 580-600, 2017.

[6] S. B. Schorr and A. M. Okamura, "Fingertip tactile devices for virtual object manipulation and exploration," in Proc. of CHI, 2017, pp. 31153119.

[7] D. Leonardis, M. Solazzi, I. Bortone, and A. Frisoli, "A 3-RSR haptic wearable device for rendering fingertip contact forces," IEEE Trans. Haptics, vol. 10, no. 3, pp. 305-316, 2016.

[8] M. Maisto, C. Pacchierotti, F. Chinello, G. Salvietti, A. De Luca, and D. Prattichizzo, "Evaluation of wearable haptic systems for the fingers in augmented reality applications," IEEE Trans. Haptics, vol. 10, no. 4 pp. 511-522, 2017.

[9] F. Chinello, C. Pacchierotti, M. Malvezzi, and D. Prattichizzo, "A three revolute-revolute-spherical wearable fingertip cutaneous device for stiffness rendering," IEEE Trans. Haptics, 2017.

[10] H. Kim, M. Kim, and W. Lee, "Hapthimble: A wearable haptic device towards usable virtual touch screen," in Proc. CHI, 2016, pp. 3694-3705.

[11] S. Je, B. Rooney, L. Chan, and A. Bianchi, "tactoring: A skin-drag discrete display," in Proc. CHI, 2017, pp. 3106-3114.

[12] M. Gabardi, M. Solazzi, D. Leonardis, and A. Frisoli, "A new wearable fingertip haptic interface for the rendering of virtual shapes and surface features," in Proc. IEEE Haptics Symposium, 2016, pp. 140-146.

[13] H. Benko, C. Holz, M. Sinclair, and E. Ofek, "NormalTouch and TextureTouch: High-fidelity 3D haptic shape rendering on handheld virtual reality controllers," in Proc. Annual Symposium on User Interface Software and Technology, 2016, pp. 717-728.

[14] E. Whitmire, H. Benko, C. Holz, E. Ofek, and M. Sinclair, "Haptic revolver: Touch, shear, texture, and shape rendering on a reconfigurable virtual reality controller," in Proc. CHI, 2018, p. 86.

[15] A. Lécuyer, "Simulating haptic feedback using vision: A survey of research and applications of pseudo-haptic feedback," Presence: Teleoperators and Virtual Environments, vol. 18, no. 1, pp. 39-53, 2009.

[16] I. Jang and D. Lee, "On utilizing pseudo-haptics for cutaneous fingertip haptic device," in Proc. of IEEE Haptics Symposium, 2014, pp. 635-639.

[17] M. Harders, G. Bianchi, B. Knoerlein, and G. Székely, "Calibration, registration, and synchronization for high precision augmented reality haptics," IEEE Trans. Visualization and Computer Graphics, vol. 15, no. 1, pp. 138-149, 2009.

[18] H. Culbertson and K. Kuchenbecker, "Ungrounded haptic augmented reality system for displaying roughness and friction," IEEE/ASME Trans. Mechatronics, vol. 22, no. 4, pp. 1839-1849, 2017.

[19] S. Jeon and S. Choi, "Haptic augmented reality: Taxonomy and an example of stiffness modulation," Presence: Teleoperators and Virtual Environments, vol. 18, no. 5, pp. 387-408, 2009.

[20] H. Ando, E. Kusachi, and J. Watanabe, "Nail-mounted tactile display for boundary/texture augmentation," in Proc. International Conference on Advances in Computer Entertainment Technology, 2007, pp. 292-293.

[21] T. Maeda, R. Peiris, M. Nakatani, Y. Tanaka, and K. Minamizawa, "Wearable haptic augmentation system using skin vibration sensor," in Proc. Virtual Reality International Conference, 2016, p. 25.

[22] S. Asano, S. Okamoto, and Y. Yamada, "Vibrotactile stimulation to increase and decrease texture roughness," IEEE Trans. Human-Machine Systems, vol. 45, no. 3, pp. 393-398, 2015.

[23] X. De Tinguy, C. Pacchierotti, M. Marchal, and A. Lécuyer, "Enhancing the stiffness perception of tangible objects in mixed reality using wearable haptics," in Proc. IEEE Conf. Virtual Reality and 3D User Interfaces (VR), 2018, pp. 81-90.

[24] C. Pacchierotti, G. Salvietti, I. Hussain, L. Meli, and D. Prattichizzo, "The hRing: a wearable haptic device to avoid occlusions in hand tracking," in Proc. IEEE Haptics Symposium, 2016.

[25] A. B. Vallbo, R. S. Johansson, et al., "Properties of cutaneous mechanoreceptors in the human hand related to touch sensation," Hum Neurobiol, vol. 3, no. 1, pp. 3-14, 1984.

[26] W. M. B. Tiest and A. M. Kappers, "Cues for haptic perception of compliance," IEEE Trans. Haptics, vol. 2, no. 4, pp. 189-199, 2009.

[27] H. Culbertson, J. M. Walker, and A. M. Okamura, "Modeling and design of asymmetric vibrations to induce ungrounded pulling sensation through asymmetric skin displacement," in Proc. IEEE Haptics Symposium, 2016, pp. 27-33.

[28] W. R. Provancher and N. D. Sylvester, "Fingerpad skin stretch increases the perception of virtual friction," IEEE Transactions on Haptics, vol. 2 , no. 4, pp. 212-223, 2009. 